\title{
Semiclassical theory of lasing in photonic crystals
}

\author{
Lucia Florescu \\ Department of Physics, University of Toronto, 60 St. George Street, Toronto, Ontario, Canada M5S 1A7 \\ Kurt Busch \\ Institut für Theorie der Kondensierten Materie, Universität Karlsruhe, 76128 Karlsruhe, Germany
}

Sajeev John

Department of Physics, University of Toronto, 60 St. George Street, Toronto, Ontario, Canada M5S 1A7

Received December 15, 2001; revised manuscript received March 14, 2002; accepted March 14, 2002

\begin{abstract}
We present a theoretical analysis of laser action within the bands of propagating modes of a photonic crystal. Using Bloch functions as carrier waves in conjunction with a multiscale analysis, we derive the generalized Maxwell-Bloch equations for an incoherently pumped atomic system in interaction with the electromagnetic reservoir of a photonic crystal. These general Maxwell-Bloch equations are similar to the conventional semiclassical laser equations but contain effective parameters that depend on the band structure of the linear photonic crystal. Through an investigation of steady-state laser behavior, we show that, near a photonic band edge, the rate of stimulated emission may be enhanced and the internal losses are reduced, which leads to an important lowering of the laser threshold. In addition, we find an increase of the laser output along with an additional narrowing of the linewidth at a photonic band edge. (C) 2002 Optical Society of America

OCIS codes: $140.3430,140.3490,160.3380$.
\end{abstract}

\section{INTRODUCTION}

Recent advances in material science have sparked tremendous growth in the field of photonic crystals. ${ }^{1}$ These novel optical materials consist of periodic arrays of dielectric material exhibiting strong Bragg scattering of electromagnetic waves, which in certain cases leads to the formation of a photonic bandgap (PBG). Under suitable circumstances with respect to material composition, topology, and lattice symmetry, forbidden frequency ranges can be created over which ordinary propagation of electromagnetic radiation is absent irrespective of the direction of propagation, while, at the same time, the material is nonabsorbing. The potential of these PBGs for realizing strong photon localization ${ }^{2-4}$ and the associated complete inhibition of spontaneous emission ${ }^{5,6}$ have ignited the imaginations of scientists and engineers worldwide. This ability to tailor electromagnetic dispersion relations and the associated photonic mode structures through suitably engineered photonic crystals facilitates a new approach to applications such as low-threshold, highefficiency microlasers, high-modulation-speed laser systems, ${ }^{7,8}$ ultrafast all-optical switches, ${ }^{9,10}$ all-optical microtransistors, ${ }^{11}$ and the integration of such devices onto an optical microchip. Lasers operating near a threedimensional (3-D) photonic band edge are expected to possess low-input-power thresholds as well as unusual spectral and statistical properties because of the nonMarkovian radiative dynamics of the light emitters. ${ }^{12}$ More generally, lasing is assisted by distributed feedback (DFB) in photonic crystals in all directions. For instance, low-threshold laser action at photonic stop gaps as a result of DFB gain enhancement was demonstrated for one-dimensional $^{13,14}$ (1-D) and two-dimensional ${ }^{15-19}$ (2-D) DFB structures.

Several theoretical models relevant to laser action in photonic crystals were developed recently. An analysis of pulse propagation in a $1-\mathrm{D}$ periodic dielectric structure ${ }^{20}$ predicted a large increase in the effective gain as a result of the small group velocities of the electromagnetic modes near the photonic stop gap. Similarly, studies of light amplification in 2-D gain-modulated structures (modeled as systems with complex refractive index ${ }^{21}$ ) indicated that the reduction in the group velocity near photonic stop gaps can lead to optical gain enhancement. Estimates for the lasing threshold that are based on the enhancement of electric fields in photonic crystals were derived $^{22}$ and supplemented by numerical studies. Recently, the influence of the photonic bands, lattice structure, and orientation of a $2-D$ photonic crystal on the gain enhancement and the threshold gain was investigated numerically by use of a scattering-matrix method. ${ }^{23} \mathrm{Al}-$ though these studies unveiled valuable information on lasing action in photonic crystals, they are either confined to $1-\mathrm{D}^{20}$ or linear systems $\mathrm{s}^{21}$ or rely heavily on numerical simulations, ${ }^{22,23}$ which permit only limited insight into the underlying physical processes.

In the present paper, we develop a semiclassical theory of laser action in photonic crystals by carrying out a multiscale analysis of the appropriate Maxwell-Bloch equations. This approach allows us to derive expressions for gain- and saturation-enhancement factors as well as for the cavity losses of finite-sized systems that can readily be evaluated from realistic photonic band structure calculation. We discuss the influence of these effective param- 
eters on the laser threshold, input-output characteristics, and the laser linewidth. Furthermore, this semiclassical model of laser action in photonic crystals that we present may represent a starting point for studies of novel phenomena related to non-Markovian radiative effects near a true PBG.

The paper is organized as follows: In Section 2, we derive the equation of motion for pulses propagating in photonic crystals and interacting with an incoherently pumped atomic system. In Section 3, we investigate the lasing threshold, the input-output characteristics, and the associated laser linewidth. Finally, in Section 4, we discuss the results and the possible generalizations of the model developed here.

\section{SEMICLASSICAL PHOTONIC-CRYSTAL LASER EQUATIONS}

\section{A. Model}

We consider a periodic dielectric medium doped with resonant two-level atoms. For intense optical pulses containing many photons a semiclassical treatment of the radiation field is adequate. The coupled-atom-field system is then described with the Maxwell-Bloch equations. ${ }^{24}$ In this paper, we focus on band-edge lasers in 1-D and 2-D photonic crystals, but the extension to 3-D PBG materials is straightforward. In the $1-\mathrm{D}$ case, we assume that the electromagnetic wave propagates parallel to the stacking direction of the dielectric layers that make up the photonic crystals, whereas, in the 2 -D case, we assume that the electromagnetic wave propagates in the plane of periodicity with the electric field polarized perpendicular to this plane ( $E$ polarization). In both cases the propagation of electromagnetic radiation through the nonlinear optical medium is described by the scalar wave equation

$$
\begin{aligned}
\nabla^{2} E(\mathbf{x}, t)-\frac{\epsilon(\mathbf{x})}{c^{2}} \frac{\partial^{2} E(\mathbf{x}, t)}{\partial t^{2}}- & \frac{4 \pi \widetilde{\sigma}(\mathbf{x})}{c^{2}} \frac{\partial E(\mathbf{x}, t)}{\partial t} \\
& =\frac{4 \pi}{c^{2}} \frac{\partial^{2} P_{\mathrm{nl}}^{\text {atoms }}(\mathbf{x}, t)}{\partial t^{2}},
\end{aligned}
$$

where $\epsilon(\mathbf{x})$ is the periodic dielectric function describing the linear polarization effects of the photonic crystal and $P_{\mathrm{nl}}^{\mathrm{atoms}}(\mathbf{x}, t)$ is the nonlinear macroscopic polarization density of the medium that is due to the presence of resonant two-level atoms. The losses caused by background absorption resulting from other nonresonant atoms in the system are described by the phenomenological conductivity $\widetilde{\sigma}(\mathbf{x})$, which can also be adjusted to include damping caused by diffraction and cavity losses, as discussed below.

The polarization $P_{\mathrm{nl}}^{\text {atoms }}$ induced by the electromagnetic field in the active medium is decomposed into single-atom contributions

$$
P_{\mathrm{nl}}^{\text {atoms }}(\mathbf{x}, t)=\sum_{a} \delta\left(\mathbf{x}-\mathbf{x}_{a}\right) P_{a}(t)=n(\mathbf{x}) \widetilde{P}(\mathbf{x}, t),
$$

where the sum runs over the laser active atoms that are embedded in the photonic crystal at sites $\mathbf{x}_{a}, P_{a}(t)$ is the atomic polarization, $\widetilde{P}(\mathbf{x}, t)$ denotes the atomic polarization density at position $\mathbf{x}$, and $n(\mathbf{x})$ is a dimensionless weight function that characterizes the distribution of active material within the photonic crystal. For instance, $n(\mathbf{x})=1$ for a uniform distribution of the two-level atoms. For simplicity, we confine our analysis to a homogeneously broadened atomic line, although the formalism presented here can easily be extended to include an inhomogeneous broadening of the atomic line. ${ }^{24}$

Before proceeding, we analyze the quantities that enter wave equation (1) to facilitate its subsequent approximate solution. For any realistic laser system the loss term in Eq. (1) is significantly smaller than the first two terms on the left-hand side (1.h.s.) of Eq. (1) that describe the free evolution of the electromagnetic field at optical frequencies $\omega$. In practice, background absorption, scattering, or diffraction losses cause extinction of the wave on a length scale $^{24}$ of $l_{e} \approx 10 \mathrm{~cm} \div 1 \mathrm{~m}$ (which is much longer compared with the optical wavelength of $\lambda \approx 10^{-6} \mathrm{~m}$ ). Similarly, the driving term on the right-hand side (r.h.s.) of Eq. (1) is typically much smaller than the first two terms on the 1.h.s. of Eq. (1). The atomic polarization density, which is coherently induced by the cavity-mode field $E(\mathbf{x}, t)$, is given by the constitutive relation $\widetilde{P}(\mathbf{x}, t)$ $\simeq \chi E(\mathbf{x}, t)$. For typical densities of resonant impurity atoms $\left(10^{24}-10^{25} \mathrm{~m}^{-3}\right)$ in the linear regime the optical susceptibility $\chi$ is of the order of ${ }^{24} 10^{-8}$. We can make explicit these different scales through the introduction of a small dimensionless parameter $\mu \ll 1\left(\mu \approx \lambda / l_{e} \approx \chi\right.$ $\approx 10^{-6}$ or less) by rewriting the conductivity $\widetilde{\sigma}(\mathbf{x})$ and the atomic polarization $\widetilde{P}(\mathbf{x}, t)$ as

$$
\begin{aligned}
\widetilde{\sigma}(\mathbf{x}) & \rightarrow \mu \sigma(\mathbf{x}), \\
\widetilde{P}(\mathbf{x}, t) & \rightarrow \mu P(\mathbf{x}, t),
\end{aligned}
$$

where $\sigma(\mathbf{x})$ and $P(\mathbf{x}, t)$ are quantities comparable with $\omega \epsilon(\mathbf{x})$ and $E(\mathbf{x}, t)$, respectively. We note that, although structural imperfections may cause a decrease of the extinction length in the photonic crystal, it is still possible to consider that losses scale with the first power of the expansion parameter $\mu$, even for a decrease in the extinction length by several orders of magnitude. With these redefinitions wave equation (1) may now be written as

$$
\begin{aligned}
\nabla^{2} E(\mathbf{x}, t)-\frac{\epsilon(\mathbf{x})}{c^{2}} \frac{\partial^{2} E(\mathbf{x}, t)}{\partial t^{2}} & -\mu \frac{4 \pi \sigma(\mathbf{x})}{c^{2}} \frac{\partial E(\mathbf{x}, t)}{\partial t} \\
& =\mu \frac{4 \pi}{c^{2}} n(\mathbf{x}) \frac{\partial^{2} P(\mathbf{x}, t)}{\partial t^{2}} .
\end{aligned}
$$

We now consider the atomic Bloch equations for the periodic dielectric structure doped with resonant atoms. The time evolution of the single-atom polarization $P_{a}(t)$ is described through ${ }^{24}$

$$
\frac{\mathrm{d}^{2} P_{a}(t)}{\mathrm{d} t^{2}}+2 \widetilde{\gamma}_{\perp} \frac{\mathrm{d} P_{a}(t)}{\mathrm{d} t}+\omega_{a}^{2} P_{a}(t)=-2 \omega_{a} \widetilde{\Omega}\left|\mathbf{d}_{12}\right| \Delta N_{a}(t) .
$$

Here $\tilde{\gamma}_{\perp}$ is the dephasing rate of the atomic dipole moment, $\omega_{a}$ is the resonant atomic frequency, and $\mathbf{d}_{12}$ is the dipole matrix element of the atomic transition. In the atomic Bloch equation (6), we introduced the so-called instantaneous complex Rabi frequency 


$$
\widetilde{\Omega} \equiv \frac{\left|\mathbf{d}_{12}\right| E\left(\mathbf{x}_{a}, t\right)}{\hbar}
$$

as a measure of the strength of the interaction between the driving field and the atomic transition. Finally, the saturable nonlinearity responsible for lasing action appears in the driving term that contains the atomicpopulation inversion $\Delta N_{a}(t) \equiv N_{2, a}(t)-N_{1, a}(t)$, where $N_{2, a}(t)$ and $N_{1, a}(t)$ are the excited- and the ground-state atomic populations, respectively. This atomic-population inversion is, in turn, driven (on a time scale much longer than the optical period $2 \pi / \omega$ ) through radiative emission and relaxation processes as well as through incoherent pumping of the atomic system ${ }^{24}$

$$
\frac{\mathrm{d} \Delta N_{a}(t)}{\mathrm{d} t}=\widetilde{\gamma}_{||}\left[\Delta N_{\mathrm{eq}, a}-\Delta N_{a}(t)\right]+2 \frac{\widetilde{\Omega}}{\omega_{a}} \frac{1}{\left|\mathbf{d}_{12}\right|} \frac{\mathrm{d} P_{a}(t)}{\mathrm{d} t},
$$

where $\widetilde{\gamma}_{\|}$is the decay rate of the atomic upper level, $\Delta N_{\text {eq, } a}$ is the steady-state equilibrium inversion, and the final term describes stimulated emission. Here we assume that the local electromagnetic density of states (LDOS) at position $\mathbf{x}$ (into which the atoms emit radiation) varies slowly with frequency $\omega$. More specifically, we assume that the LDOS is a smooth function of frequency on the scale of $\widetilde{\gamma}_{\|}^{-1}$. In cases in which the LDOS varies considerably on the scale $\tilde{\gamma}_{\|}^{-1}$ (such as near a $3-\mathrm{D}$ photonic band edge), Eqs. (6) and (8) must be replaced by integrodifferential equations involving a non-Markovian memory kernel and nonexponential decay of the excited atomic state. ${ }^{12}$

Analogously to the analysis of wave equation (1), we introduce into the equation of motion for the atomic variables the small parameter $\mu$ to make explicit the different time scales over which they vary. In general, the rate of change of the atomic-polarization and the atomicpopulation inversions that are due to relaxation or pumping is very slow compared with the optical frequency, ${ }^{24}$ $\tilde{\gamma}_{\perp, \|} \ll \omega$ (here we assume that both relaxation and dephasing take place on a time scale of nanoseconds, such that $\left.\widetilde{\gamma}_{\perp, \|} \approx 10^{-6} \omega\right)$. Consistent with our multiple-scale analysis, we rewrite the rates $\widetilde{\gamma}_{\perp}$ and $\tilde{\gamma}_{\|}$, describing the dephasing and the relaxation processes, respectively, as

$$
\begin{gathered}
\tilde{\gamma}_{\perp} \rightarrow \mu \gamma_{\perp}, \\
\tilde{\gamma}_{\|} \rightarrow \mu \gamma_{\|},
\end{gathered}
$$

where $\gamma_{\perp}$ and $\gamma_{\|}$are now quantities comparable with the optical frequency $\omega$. Strictly speaking, there are physical situations in which the dephasing process is faster, sometimes on a picosecond scale (such as for organic dyes). It is, nevertheless, possible to consider that both dephasing and relaxation processes correspond to the same order of the expansion parameter: $\mu \approx 10^{-6}$. Further, we note that the term on the r.h.s. of Eq. (6) and the last term on the r.h.s. of Eq. (8) are both induced by atom-field interaction. The weak coupling between the atomic system and the radiation reservoir of the photonic crystal is expressed by the fact that the magnitude of the Rabi frequency equation (7) is small compared with the optical frequency. For instance, in a typical optical transition in a conventional dye laser, ${ }^{24}$ dipole moments of $\left|\mathbf{d}_{12}\right| \approx 10^{-29}-10^{-28} \mathrm{~cm}$ and electric field amplitudes of $|E| \approx 10^{5} \mathrm{~V} / \mathrm{m}$ (which corresponds to laser operation just below saturation of nonlinearity) yield $|\widetilde{\Omega}| / \omega$ $\simeq 10^{-6}-10^{-5}$. Thus the term on the r.h.s. of Eq. (6) for the atomic polarization is much smaller than the terms on the l.h.s. that describe the free oscillation

$$
\omega_{a} \widetilde{\Omega}\left|\mathbf{d}_{12}\right| \Delta N_{a}(t) \sim \omega_{a} \widetilde{\Omega} P_{a}(t) \ll \omega_{a}^{2} P_{a}(t),
$$

whereas, for the last term on the r.h.s. of the Eq. (8) of the evolution of the atomic-population inversion, we have

$$
\frac{\widetilde{\Omega}}{\omega_{a}} \frac{1}{\left|\mathbf{d}_{12}\right|} \frac{\mathrm{d} P_{a}(t)}{\mathrm{d} t} \sim \frac{\widetilde{\Omega}}{\omega_{a}} \frac{\mathrm{d} \Delta N_{a}(t)}{\mathrm{d} t} \ll \frac{\mathrm{d} \Delta N_{a}(t)}{\mathrm{d} t} .
$$

Consequently, we rewrite the Rabi frequency equation (7) as

$$
\widetilde{\Omega} \rightarrow \mu \Omega,
$$

where now $\Omega$ is comparable with $\omega$. With these redefinitions and after multiplying the atomic Bloch equations (6) and (8) by $\delta\left(\mathbf{x}-\mathbf{x}_{a}\right)$, summing up over the atoms, and recalling the definition of the Rabi frequency equation (7), we obtain the equation of motion for the atomicpolarization and the atomic-population inversion densities

$$
\begin{aligned}
\frac{\mathrm{d}^{2} P(\mathbf{x}, t)}{\mathrm{d} t^{2}}+\mu\left[2 \gamma_{\perp} \frac{\mathrm{d} P(\mathbf{x}, t)}{\mathrm{d} t}\right]+\omega_{a}^{2} P(\mathbf{x}, t) & \\
= & \mu\left[-2 \omega_{a} \frac{\left|\mathbf{d}_{12}\right|^{2}}{\hbar} E(\mathbf{x}, t) \Delta N(\mathbf{x}, t)\right], \\
\frac{\mathrm{d} \Delta N(\mathbf{x}, t)}{\mathrm{d} t}=\mu\left[R-\gamma_{\|} \Delta N(\mathbf{x}, t)\right. & \\
& \left.+\frac{2}{\hbar \omega_{a}} E(\mathbf{x}, t) \frac{\mathrm{d} P(\mathbf{x}, t)}{\mathrm{d} t}\right] .
\end{aligned}
$$

Here $\Delta N(\mathbf{x}, t)$ is the atomic-population inversion density, and $R \equiv \gamma_{\|} \Delta N_{\text {eq }}$ is the rate at which the atoms are incoherently pumped from the ground state to the excited state $\left(\Delta N_{\text {eq }}\right.$ is the homogeneous equilibrium populationinversion density). Furthermore, it should be noted that Eqs. (14) and (15) are valid only at positions $\mathbf{x}$, where the dimensionless distribution function $n(\mathbf{x})$ of the active material is different from zero.

Equations (5) and (14) (and their complex conjugates) together with Eq. (15) constitute the basic semiclassical equations for electromagnetic waves in photonic crystals interacting with a collection of incoherently pumped twolevel atoms. The general solutions of Eqs. (5), (14), and (15) represent formidable tasks. In what follows, we develop an approximate solution that is analogous to the slowly varying envelope approximation of standard laser resonators. ${ }^{24}$

\section{B. Multiscale Analysis}

The key simplification of Eqs. (5), (14), and (15) arises from separating fast from slow variations in space and time in the electromagnetic field $E(\mathbf{x}, t)$, the atomicpolarization density $P(\mathbf{x}, t)$, and the atomic-population inversion density $\Delta N(\mathbf{x}, t)$. This separation is facili- 
tated by the presence of the small parameter $\mu$ (which, according to the above discussion of typical physical situations, we note is roughly $10^{-6}$ in each case). In our multiple-scale analysis, ${ }^{25}$ we replace the space and the time variables, $\mathbf{x}$ and $t$, respectively, with a new set of independent variables, $\mathbf{x}_{n} \equiv \mu^{n} \mathbf{x}$ and $t_{n} \equiv \mu^{n} t$, respectively. This replacement is accompanied by a corresponding expansion of the appropriate functions in powers of the parameter $\mu$ to construct a hierarchy of equations that effectively separate the different scales of the problem. In the present case, the fastest spatial scale $\mathbf{x}_{0}$ corresponds to the wavelength of electromagnetic waves propagating in the photonic crystal, and the fastest temporal scale $t_{0}$ is associated with the optical period $2 \pi / \omega$. In terms of the new coordinates the spatial and the temporal derivatives are then rewritten as

$$
\begin{gathered}
\frac{\partial}{\partial \mathbf{x}}=\frac{\partial}{\partial \mathbf{x}_{0}}+\mu \frac{\partial}{\partial \mathbf{x}_{1}}+\mu^{2} \frac{\partial}{\partial \mathbf{x}_{2}}+\ldots, \\
\frac{\partial}{\partial t}=\frac{\partial}{\partial t_{0}}+\mu \frac{\partial}{\partial t_{1}}+\mu^{2} \frac{\partial}{\partial t_{2}}+\ldots,
\end{gathered}
$$

from which expressions for higher derivatives can be readily obtained. In addition, we introduce a hierarchy of contributions to the field and the atomic variables

$$
\begin{aligned}
E(\mathbf{x}, t) & =E^{(0)}+\mu E^{(1)}+\mu^{2} E^{(2)}+\ldots, \\
P(\mathbf{x}, t) & =P^{(0)}+\mu P^{(1)}+\mu^{2} P^{(2)}+\ldots, \\
\Delta N(\mathbf{x}, t) & =\Delta N^{(0)}+\mu \Delta N^{(1)}+\mu^{2} \Delta N^{(2)}+\ldots,
\end{aligned}
$$

where the electromagnetic field $E^{(n)}=E^{(n)}\left(\mathbf{x}_{0}\right.$, $\left.\mathbf{x}_{1}, \ldots ; t_{0}, t_{1}, \ldots\right)$ as well as the atomic polarization $P^{(n)}$ $=P^{(n)}\left(\mathbf{x}_{0}, \mathbf{x}_{1}, \ldots ; t_{0}, t_{1}, \ldots\right)$ vary on all spatial and temporal scales $\mathbf{x}_{n}$ and $t_{n}$. However, although the atomic inversion $\Delta N$ varies on all spatial scales $\mathbf{x}_{n}$, we assume that it does not vary on the time scale $t_{0}$ of the optical period. Transitions between the upper and the lower atomic levels do not occur on the optical time scale, a fact that is manifested through the presence of the small parameter $\mu$ on the r.h.s. of Eq. (15). Consequently, we make the ansatz $\Delta N^{(n)}=\Delta N^{(n)}$ $\times\left(\mathbf{x}_{0}, \mathbf{x}_{1}, \ldots ; t_{1}, t_{2}, \ldots\right)$. In addition, we assume that the periodic dielectric function $\epsilon(\mathbf{x})=\epsilon\left(\mathbf{x}_{0}\right)$, the conductivity $\sigma(\mathbf{x})=\sigma\left(\mathbf{x}_{0}\right)$, and the atomic-distribution function $n(\mathbf{x})=n\left(\mathbf{x}_{0}\right)$ vary exclusively on the smallest length scale $\mathbf{x}_{0}$.

To generate the hierarchy of equations that reflect the different scales involved in the problem, we insert expansion equations (18), (19), and (20) into motion equations (5), (14), and (15), respectively, expand the time and the space derivatives according to Eqs. (16) and (17), and collect terms with equal powers of $\mu$. As expected, the system's behavior on the fastest scale (zeroth order in $\mu$ ) is determined solely through the linear properties of the photonic crystal (for the electromagnetic field) and the atomic polarization (free oscillation of the atoms). We obtain

$$
\left[\frac{\partial^{2}}{\partial \mathbf{x}_{0}^{2}}-\frac{\epsilon\left(\mathbf{x}_{0}\right)}{c^{2}} \frac{\partial^{2}}{\partial t_{0}^{2}}\right] E^{(0)}=0,
$$

$$
\frac{\partial^{2} P^{(0)}}{\partial t_{0}^{2}}+\omega_{a}^{2} P^{(0)}=0 .
$$

Because the nonlinear effects occur on longer time scales, Eqs. (21) and (22) suggest a decomposition of $E^{(0)}$ and $P^{(0)}$ into carrier waves that are given by the set of eigenfunctions $\left\{\Phi_{m}\left(\mathbf{x}_{0}\right)\right\}$ of the cold cavity and the associated slowly varying envelope functions $\mathcal{E}$ and $\mathcal{P}$. For simplicity, we consider situations in which one mode is dominant and make the ansatz

$$
\begin{aligned}
E^{(0)} & \left(\mathbf{x}_{0}, \mathbf{x}_{1}, \ldots ; t_{0}, t_{1}, \ldots\right) \\
& =\mathcal{E}\left(\mathbf{x}_{1}, \mathbf{x}_{2}, \ldots ; t_{1}, t_{2}, \ldots\right) \Phi_{m}\left(\mathbf{x}_{0}\right) \exp \left(-i \omega_{m} t_{0}\right)+\text { c.c. }
\end{aligned}
$$

$$
\begin{aligned}
P^{(0)} & \left(\mathbf{x}_{0}, \mathbf{x}_{1}, \ldots ; t_{0}, t_{1}, \ldots\right) \\
& =\mathcal{P}\left(\mathbf{x}_{1}, \mathbf{x}_{2}, \ldots ; t_{1}, t_{2}, \ldots\right) \Phi_{m}\left(\mathbf{x}_{0}\right) \exp \left(-i \omega_{m} t_{0}\right)+\text { c.c.. }
\end{aligned}
$$

The eigenfunctions $\Phi_{m}\left(\mathbf{x}_{0}\right)$ and the associated eigenvalues $\omega_{m}$ of the photonic crystal are given by the solutions of the homogeneous wave equation

$$
\left[\frac{\partial^{2}}{\partial \mathbf{x}_{0}^{2}}+\frac{\omega_{m}^{2}}{c^{2}} \epsilon\left(\mathbf{x}_{0}\right)\right] \Phi_{m}\left(\mathbf{x}_{0}\right)=0
$$

and satisfy the orthogonality relations

$$
\int_{\text {cell }} \Phi_{m}^{*}\left(\mathbf{x}_{0}\right) \epsilon\left(\mathbf{x}_{0}\right) \Phi_{m^{\prime}}\left(\mathbf{x}_{0}\right) \mathrm{d} \mathbf{x}_{0}=\delta_{m, m^{\prime}}
$$

Combining Eq. (24) with Eq. (22), we obtain the requirement that the atomic transition should be contained in the spectrum of eigenfrequencies of the cold cavity:

$$
\omega_{m}=\omega_{a} \text {. }
$$

Nonlinear interaction effects between the electromagnetic field propagating in the photonic crystal and the resonant two-level atoms occur on the slow scale represented by the terms of first order in $\mu$ :

$$
\begin{gathered}
{\left[-c^{2} \frac{\partial^{2}}{\partial \mathbf{x}_{0}^{2}}+\epsilon\left(\mathbf{x}_{0}\right) \frac{\partial^{2}}{\partial t_{0}^{2}}\right] E^{(1)}} \\
=\left[2 c^{2} \frac{\partial}{\partial \mathbf{x}_{0}} \frac{\partial}{\partial \mathbf{x}_{1}}-2 \epsilon\left(\mathbf{x}_{0}\right) \frac{\partial}{\partial t_{0}} \frac{\partial}{\partial t_{1}}+4 \pi \sigma\left(\mathbf{x}_{0}\right) \frac{\partial}{\partial t_{0}}\right] E^{(0)} \\
+4 \pi n\left(\mathbf{x}_{0}\right) \frac{\partial^{2}}{\partial t_{0}^{2}} P^{(0)}, \\
\left(\frac{\partial^{2}}{\partial t_{0}^{2}}+\omega_{a}^{2}\right) P^{(1)}=-2\left(\frac{\partial^{2}}{\partial t_{0} \partial t_{1}}+\gamma_{\perp} \frac{\partial}{\partial t_{0}}\right) P^{(0)} \\
\quad-2 \omega_{a} \frac{\left|\mathbf{d}_{12}\right|^{2}}{\hbar} E^{(0)} \Delta N^{(0)}, \\
\frac{\partial}{\partial t_{1}} \Delta N^{(0)}=R-\gamma_{\|} \Delta N^{(0)}+\frac{2}{\hbar \omega_{a}} E^{(0)} \frac{\partial P^{(0)}}{\partial t_{0}} .
\end{gathered}
$$

Because to the lowest order, the electromagnetic field as well as the atomic polarization are dominated by the 
mode $\Phi_{m}\left(\mathbf{x}_{0}\right)$ [see Eqs. (21) and (22)], we have to include all but this mode in the ansatz for the higher-order corrections $E^{(1)}$ and $P^{(1)}$ :

$$
\begin{aligned}
& E^{(1)}\left(\mathbf{x}_{0}, \mathbf{x}_{1}, \ldots ; t_{0}, t_{1}, \ldots\right) \\
& =\sum_{l \neq m} e\left(\mathbf{x}_{1}, \mathbf{x}_{2}, \ldots ; t_{1}, t_{2}, \ldots\right) \Phi_{l}\left(\mathbf{x}_{0}\right) \exp \left(-i \omega_{m} t_{0}\right)+\text { c.c. },
\end{aligned}
$$

$$
\begin{aligned}
& P^{(1)}\left(\mathbf{x}_{0}, \mathbf{x}_{1}, \ldots ; t_{0}, t_{1}, \ldots\right) \\
& =\sum_{l \neq m} p\left(\mathbf{x}_{1}, \mathbf{x}_{2}, \ldots ; t_{1}, t_{2}, \ldots\right) \Phi_{l}\left(\mathbf{x}_{0}\right) \exp \left(-i \omega_{m} t_{0}\right)+\text { c.c. }
\end{aligned}
$$

To obtain the equations of motion for the physically relevant envelope functions $\mathcal{E}$ and $\mathcal{P}$, we insert expansion equations (23), (24), (31), and (32) into Eqs. (28), (29), and (30) that describe the leading order of the atom-field interaction, use the frequency-resonance condition (27), and project the resulting system of equations onto the subspace spanned by the dominant mode $\Phi_{m}$. We obtain

$$
\begin{aligned}
\mathbf{v}_{m} \cdot \nabla \mathcal{E}(\mathbf{x}, t) & +\frac{\partial \mathcal{E}(\mathbf{x}, t)}{\partial t}+2 \pi \sigma_{m} \mathcal{E}(\mathbf{x}, t) \\
& =2 \pi i \alpha_{m} \omega_{m} \mathcal{P}(\mathbf{x}, t),
\end{aligned}
$$

$$
\begin{aligned}
\frac{\partial \mathcal{P}(\mathbf{x}, t)}{\partial t}+\gamma_{\perp} \mathcal{P}(\mathbf{x}, t) & \\
& =-i \frac{\left|\mathbf{d}_{12}\right|^{2}}{\hbar} \mathcal{E}(\mathbf{x}, t) \widetilde{\Delta N}(\mathbf{x}, t),
\end{aligned}
$$

$$
\begin{aligned}
\frac{\partial \widetilde{\Delta N}(\mathbf{x}, t)}{\partial t}= & R-\gamma_{\|} \widetilde{\Delta N}(\mathbf{x}, t)+\frac{2 i}{\hbar} \beta_{m}\left[\mathcal{E}(\mathbf{x}, t) \mathcal{P}^{*}(\mathbf{x}, t)\right. \\
& \left.-\mathcal{P}(\mathbf{x}, t) \mathcal{E}^{*}(\mathbf{x}, t)\right]
\end{aligned}
$$

where the quantities $\mathbf{v}_{m}, \sigma_{m}, \alpha_{m}, \widetilde{\Delta N}$, and $\beta_{m}$ are defined below. Here we neglected rapidly oscillating terms that are proportional to $\exp \left( \pm 2 i \omega_{m} t_{0}\right)$ (rotating wave approximation), truncated the multiscale hierarchy on the scales of $\mathbf{x}_{1}$ and $t_{1}$, and dropped the corresponding subscripts such that $\mathbf{x} \equiv \mathbf{x}_{1}$ and $t \equiv t_{1}$. Higher-order corrections to the dominant order in the atom-field interaction can be incorporated in a systematic fashion by one's carrying the multiscale analysis to correspondingly higher orders.

Equations (33), (34), and (35) contain a number of quantities that contain the averaged information on the fast scale, which is of relevance to the buildup of laser intensity on the slow scale. First, a pulse with a carrier wave $\Phi_{m}\left(\mathbf{x}_{0}\right)$ propagates in a photonic crystal with the group velocity $\mathbf{v}_{m},{ }^{26-28}$ where

$$
\mathbf{v}_{m}=\frac{c^{2}}{\omega_{m}} \int_{\text {cell }} \phi_{m}^{*}\left(\mathbf{x}_{0}\right)\left(-i \frac{\mathrm{d}}{\mathrm{d} \mathbf{x}_{0}}\right) \Phi_{m}\left(\mathbf{x}_{0}\right) \mathrm{d} \mathbf{x}_{0} .
$$

Second, the carrier wave [Bloch function $\Phi_{m}\left(\mathbf{x}_{0}\right)$ ], which exhibits a spatial structure that is different from that of a plane wave, must overlap the distribution $n\left(\mathbf{x}_{0}\right)$ of the ac- tive material within the photonic crystal's unit cell to contribute to the effective atomic-population inversion, $\widetilde{\Delta N}(\mathbf{x}, t)$, defined by

$$
\widetilde{\Delta N}(\mathbf{x}, t) \equiv \frac{\int_{\text {cell }} \Delta N\left(\mathbf{x}_{0}, \mathbf{x}_{1} ; t_{1}\right) x\left|\Phi_{m}\left(\mathbf{x}_{0}\right)\right|^{2} n\left(\mathbf{x}_{0}\right) \mathrm{d} \mathbf{x}_{0}}{\int_{\text {cell }}\left|\Phi_{m}\left(\mathbf{x}_{0}\right)\right|^{2} n\left(\mathbf{x}_{0}\right) \mathrm{d} \mathbf{x}_{0}} .
$$

Likewise, the overlap of strong fields from the Bloch wave with the atomic distribution leads to an effective fieldenhancement factor $\alpha_{m}$ for stimulated emission, given by

$$
\alpha_{m}=\int_{\text {cell }}\left|\Phi_{m}\left(\mathbf{x}_{0}\right)\right|^{2} n\left(\mathbf{x}_{0}\right) \mathrm{d} \mathbf{x}_{0},
$$

and an enhancement of the saturable nonlinear response expressed through the effective nonlinear couplingenhancement factor $\beta_{m}$,

$$
\beta_{m}=\frac{\int_{\text {cell }}\left|\Phi_{m}\left(\mathbf{x}_{0}\right)\right|^{4} n\left(\mathbf{x}_{0}\right) \mathrm{d} \mathbf{x}_{0}}{\int_{\text {cell }}\left|\Phi_{m}\left(\mathbf{x}_{0}\right)\right|^{2} n\left(\mathbf{x}_{0}\right) \mathrm{d} \mathbf{x}_{0}} .
$$

In a similar manner the effective extinction parameter $\sigma_{m}$ is given by

$$
\sigma_{m}=\int_{\text {cell }}\left|\Phi_{m}\left(\mathbf{x}_{0}\right)\right|^{2} \sigma\left(\mathbf{x}_{0}\right) \mathrm{d} \mathbf{x}_{0} .
$$

Equations (33)-(35) together with expressions (36)(40) constitute the central result of our semiclassical analysis of laser action in photonic crystals. They are the generalization of the semiclassical equations for conventional lasers, ${ }^{24}$ where the effects associated with the radiation reservoir of the photonic crystal manifest themselves in the presence of the group velocity $\mathbf{v}_{m}$, the gainenhancement factor $\alpha_{m}$, and the saturation factor $\beta_{m}$, which may all be obtained from photonic band structure computation. We note that these quantities reduce to those of a conventional laser when the dielectric function is set to a constant and the carrier waves are replaced by plane waves. Furthermore, we point out that, within our model, the time evolution of the atomic polarization is not directly affected by the characteristics of the photonic crystal, except possibly through an overall modification of the decay parameters $\gamma_{\|}$and $\gamma_{\perp}$, related to the modified coupling of atoms to nonlasing modes. Primarily, the photonic crystal has an indirect effect on the atomic polarization through the atomic-population inversion and the electromagnetic field entering its equation of motion (34). More direct effects on the atomic polarization and inversion may arise if the periodic modulation of the dielectric constant $\epsilon(\mathbf{x})$ is sufficiently strong to cause rapid variation of the LDOS with frequency. ${ }^{12}$

\section{Adiabatic Elimination of Atomic Polarization}

For the physically most relevant situation of pulses that are longer than the polarization-relaxation time, it is well justified to assume a quasi-equilibrium situation and to eliminate adiabatically the polarization from the 
Maxwell-Bloch equations (33) and (35). Considering the steady-state solution of Eq. (34)

$$
\mathcal{P}(\mathbf{x}, t)=-i \frac{\left|\mathbf{d}_{12}\right|^{2}}{\hbar \gamma_{\perp}} \mathcal{E}(\mathbf{x}, t) \widetilde{\Delta N}(\mathbf{x}, t),
$$

we obtain from Eqs. (33) and (35) the reduced set of the equations of motion for the field envelope $\mathcal{E}(\mathbf{x}, t)$ and the atomic inversion $\widetilde{\delta N}(\mathbf{x}, t)$

$$
\begin{aligned}
\mathbf{v}_{m} \cdot \nabla \mathcal{E}(\mathbf{x}, t) & +\frac{\partial \mathcal{E}(\mathbf{x}, t)}{\partial t} \\
= & \frac{1}{2}\left[-\gamma_{m}+\alpha_{m} \frac{4 \pi\left|\mathbf{d}_{12}\right|^{2} \omega_{m}}{\hbar \omega_{\perp}} \widetilde{\Delta N}(\mathbf{x}, t)\right] \mathcal{E}(\mathbf{x}, t),
\end{aligned}
$$

$$
\begin{aligned}
\frac{\partial \widetilde{\Delta N}(\mathbf{x}, t)}{\partial t}= & R-\gamma_{\|} \widetilde{\Delta N}(\mathbf{x}, t) \\
& -\beta_{m} \frac{4\left|\mathbf{d}_{12}\right|^{2}}{\hbar^{2} \gamma_{\perp}}|\mathcal{E}(\mathbf{x}, t)|^{2} \widetilde{\Delta N}(\mathbf{x}, t),
\end{aligned}
$$

where we introduced the effective cavity-loss rate $\gamma_{m} \equiv 4 \pi \sigma_{m}+2 \kappa_{m}$ and where additional losses for mode $\Phi_{m}$, other than the background absorption $\sigma_{m}$, were incorporated phenomenologically through the cavityleakage term $\kappa_{m}$. In particular, cavity leakage is associated with a finite sample size or a finite pumping region of on otherwise infinite system (see Section 3, below). Equations (42) and (43) represent a convenient starting point for the study of laser oscillation within our semiclassical model of laser action in photonic crystals.

\section{STEADY-STATE CHARACTERISTICS}

Important aspects of laser action such as the threshold pump intensity, the input-output characteristics, and the laser linewidth can be determined from the steady-state or the cw operation of the laser. These are directly influenced by the effective parameters $\mathbf{v}_{m}, \alpha_{m}, \beta_{m}$, and $\gamma_{m}$.

In the cw limit, Eq. (43) can be solved for the steadystate atomic-population inversion $\overline{\Delta N}(\mathbf{x})$,

$$
\overline{\Delta N}(\mathbf{x})=\frac{R / \gamma_{\|}}{1+\beta_{m} I(\mathbf{x})},
$$

where we introduced the dimensionless intensity

$$
I(\mathbf{x})=\frac{|\mathcal{E}(\mathbf{x}, t)|^{2}}{I_{\text {sat }}},
$$

where

$$
I_{\text {sat }} \equiv \frac{\hbar^{2} \gamma_{\perp} \gamma_{\|}}{4\left|\mathbf{d}_{12}\right|^{2}}
$$

is the line-center saturation field intensity. It represents the field intensity at which the nonlinear response of the two-level atoms in our model becomes important. For $I \ll I_{\text {sat }}$, the atoms respond to an external electric field like simple harmonic oscillators, whereas, for $I \gg I_{\text {sat }}$, the atomic response is saturated. $I_{\text {sat }}$ itself may be modified from its value in free space, $I_{\text {sat }}^{0}$, by the modification of the LDOS in a photonic crystal and the coupling strength of various electromagnetic modes to the atom.

As a consequence, the field-envelope equation (42) for the steady-state case becomes

$$
\mathbf{v}_{m} \cdot \nabla \mathcal{E}(\mathbf{x})=\frac{1}{2}\left[-\gamma_{m}+\frac{\mathcal{G}_{m}}{1+\beta_{m} I(\mathbf{x})}\right] \mathcal{E}(\mathbf{x}),
$$

where $\mathcal{G}_{m}$ denotes the unsaturated gain coefficient

$$
\mathcal{G}_{m}=\frac{\pi \alpha_{m}\left(\hbar \omega_{m}\right) R}{I_{\text {sat }}} .
$$

It follows directly from Eq. (47) that there are two distinct regimes of pulse growth. For small values of the field envelope $\mathcal{E}(\mathbf{x}), \beta I(\mathbf{x})$ can be neglected. As a consequence, the envelope grows exponentially with a rate of $\left(\mathcal{G}_{m}-\gamma_{m}\right) / v_{m}$. For sufficiently large values of the field envelope a saturation regime develops such that the saturation term $\beta_{m} \mathcal{E}(\mathbf{x})$ gradually reduces to zero the effective growth rate. The limiting steady-state value of the intensity, corresponding to the spatial-saturation regime, is given by

$$
I_{\mathrm{ss}}=\frac{1}{\beta_{m}}\left(\frac{\mathcal{G}_{m}}{\gamma_{m}}-1\right) .
$$

Equation (49) suggests that amplification is possible only if $\mathcal{G}_{m} / \gamma_{m}>1$, and therefore the laser threshold condition is identified as $\mathcal{G}_{m}=\gamma_{m}$. For given material parameters this condition translates into a threshold value of the excitation rate

$$
R_{\mathrm{thr}}=\bar{I}_{\mathrm{sat}}\left(\frac{\gamma_{m}}{\gamma_{0}}\right) \frac{1}{\bar{\alpha}_{m}} R_{\mathrm{thr}}^{0},
$$

which is necessary for sustaining amplification in the photonic-crystal laser. Here $R_{\text {thr }}^{0}=\gamma_{0} I_{\text {sat }}^{0} / \int_{\text {cell }} \mathrm{d} \mathbf{x}_{0} n\left(\mathbf{x}_{0}\right)$ and $\bar{I}_{\text {sat }} \equiv I_{\text {sat }} / I_{\text {sat }}^{0}$ is the ratio of the line-center saturation field strength in the photonic crystal to that in free space, $\quad \gamma_{m}=4 \pi \int_{\text {cell }} \mathrm{d} \mathbf{x}_{0}\left|\Phi_{m}\left(\mathbf{x}_{0}\right)\right|^{2} \sigma\left(\mathbf{x}_{0}\right)+2 \kappa_{m} \quad$ is the overall cavity-loss rate in the photonic crystal, $\gamma_{0}=4 \pi \int_{\text {cell }} \mathrm{d} \mathbf{x}_{0} \sigma\left(\mathbf{x}_{0}\right)+2 \kappa_{0}$ is the corresponding cavityloss rate if the photonic crystal were replaced by a uniform dielectric medium, and

$$
\alpha_{m} \equiv \int_{\text {cell }} \mathrm{d} \mathbf{x}_{0}\left|\Phi_{m}\left(\mathbf{x}_{0}\right)\right|^{2} n\left(\mathbf{x}_{0}\right) / \int_{\text {cell }} \mathrm{d} \mathbf{x}_{0} n\left(\mathbf{x}_{0}\right) .
$$

To understand the threshold behavior of realistic photonic-crystal lasers, it is necessary to discuss the additional cavity-leakage rate $\kappa_{m}$ that is associated with mode $\Phi_{m}$ for a finite sample size or a finite pumping volume. For instance, the leakage rate for a homogeneously pumped slab of photonic crystal of length $L$ oriented along the direction $\hat{\mathbf{q}}$ may be modeled by one's regarding it as a standard Fabry-Perot resonator slab whose refractive index is replaced by an effective refractive index $n_{\text {eff }}=c /\left(\left|\hat{\mathbf{q}} \cdot \mathbf{v}_{m}\right|\right) .^{22}$ The cavity-decay rate in such a resonator is related to the time required for light to traverse the length of the cavity and mirror losses that are due to the imperfect reflectivity at the interface between the slab surface and air. In terms of the cavity quality factor $Q_{m}$ this cavity leakage is expressed as $\kappa_{m}=\omega_{m} / Q_{m}$. Further, the cavity quality factor can be written $\operatorname{as}^{29} Q_{m}=2 \pi L / t_{m} \lambda_{m}$, where $t_{m}=\ln \left(1 / R_{\text {eff }}\right)$ is the fractional loss per pass through the cavity and 
$\lambda_{m}=2 \pi \omega_{m} \quad\left(c / n_{\text {eff }}\right)$. The effective reflectivity $R_{\text {eff }}$ is given by $R_{\text {eff }}=\left(n_{\text {eff }}-1\right) /\left(n_{\text {eff }}+1\right)$ such that we obtain for the cavity-decay rate the expression $\kappa_{m}=c / n_{\text {eff }}^{L} \ln \left(n_{\text {eff }}\right.$ $\left.+1 / n_{\text {eff }}-1\right)$. Near a photonic band edge, where $n_{\text {eff }}$ $\gg 1$, this expression can be approximated by $\kappa_{m}$ $\simeq c / L\left(2 / n_{\text {eff }}^{2}\right) \sim(2 / L c)\left|\hat{\mathbf{q}} \cdot \mathbf{v}_{m}\right|^{2}$. On the other hand, for a slablike active region of thickness $L$ of an otherwise infinite photonic crystal, we estimate the rate at which energy leaves the active region through the projection of the Poynting vector $\mathbf{S}_{m} \propto \mathbf{v}_{m} I(\mathbf{x})$ onto the propagation direction $\hat{\mathbf{q}}$, resulting in a cavity-loss rate of $\kappa_{m} \simeq\left|\hat{\mathbf{q}} \cdot \mathbf{v}_{m}\right| / L$.

In both instances the cavity-leakage rate $\kappa_{m}$ depends on the mode $\Phi_{m}$ through the group velocity and therefore becomes small near a photonic band edge. If we further assume that the material losses $\sigma_{m}$ are minimal for the frequency range under consideration, we can assign the same group-velocity dependence to the entire cavity loss: $\gamma_{m} \simeq 2 \kappa_{m}$.

We now discuss the lasing threshold for a photoniccrystal laser in greater detail. First, low group velocities near a photonic stop gap for a given propagation direction may lead to low cavity losses for finite photonic-crystal samples or finite-sized pumping regions in an infinite photonic crystal. In addition, there exists an enhancement of the stimulated-emission rate that originates from a field enhancement of the cold-cavity mode of the infinite photonic crystal averaged over the distribution of active material within the unit cell. This average field enhancement in the photonic-crystal structure is given by $\bar{\alpha}_{m}$ and may be computed by use of photonic band structure theory. For the free-space case, we have $\bar{\alpha}_{m}=1$, whereas the values $\bar{\alpha}_{m}>1$ and $\bar{\alpha}_{m}<1$ signify enhanced and suppressed stimulated-emission rates, respectively.

As an illustrative example, we consider $E$-polarized radiation propagating in a $2-\mathrm{D}$ photonic crystal that consists of a square lattice (lattice constant $a$ ) of cylinders (radius of $r=0.4 a$, dielectric constant of $\epsilon_{c}=12$ ) embedded in air (dielectric constant of $\epsilon_{b}=1$ ), where the active material is uniformly distributed outside the cylinder such that $n(\mathbf{x})=|\theta(\mathbf{x})|-r$, with $\theta(x)$ the Heaviside step function. The lowest bands of this 2-D photonic crystal are shown in Fig. 1. We obtain two photonic gaps separated by the second and the third bands. Figures 2 and 3 display the behavior of the group velocity ${ }^{28} \hat{\mathbf{q}} \cdot \mathbf{v}_{m}$ and the field-enhancement factor $\bar{\alpha}_{m}$ along the high-symmetry lines of the irreducible part of the first Brillouin zone for the lowest three bands. Compared with the free-space speed of light, the group velocity for bands 2 and 3 exhibits very low values. However, near the $X$ point, only band 2 displays a substantial increase in the fieldenhancement factor $\bar{\alpha}_{m}$. This region of the photondispersion relation corresponds to an air band in which the field intensity is concentrated in the air fraction of the photonic crystal, where we assume the resonant atoms reside. In contrast, band 3 exhibits a rather unfavorable gain suppression at the $X$ point. As a consequence, for a broadband emitter such as a laser dye occupying the space between cylinders lasing action is likely to occur first near the $X$ point in the second band. This result illustrates how the design issues involved in constructing an efficient photonic-crystal laser may be systematically addressed within the semiclassical theory.
Another important aspect of Eq. (49) for the saturation intensity of photonic-crystal lasers is the dependence of the output power on the pumping rate above threshold. The input-output relation for the steady-state operation of the laser is given by

$$
I_{\text {output }}=\left\{\begin{array}{ll}
0 & \text { for } R<R_{\mathrm{thr}} \\
\frac{\pi}{\beta_{m} \gamma_{m}} \frac{\alpha_{m}\left(\hbar \omega_{m}\right)}{I_{\mathrm{sat}}}\left(R-R_{\mathrm{thr}}\right) & \text { for } R>R_{\mathrm{thr}}
\end{array},\right.
$$

The slope, $\mathrm{d} I_{\text {output }} / \mathrm{d} R$, of the input-output laser characteristic is directly proportional to $\alpha_{m} /\left(\beta_{m} \gamma_{m} I_{\text {sat }}\right)$. In a typical photonic crystal the field-enhancement factor $\alpha_{m}$ and the nonlinear coupling-enhancement factor $\beta_{m}$ exhibit similar behavior.

In Fig. 4, we display the variation of the scaled enhancement factor $\bar{\beta}_{m} \equiv \beta_{m} / \beta_{0}$, where $\beta_{0}$ $=\int_{\text {cell }} \mathrm{d} \mathbf{x}_{0} n\left(\mathbf{x}_{0}\right)$, along the high-symmetry lines of the irreducible part of the first Brillouin zone for the same system for which we computed the band structure (Fig. 1),

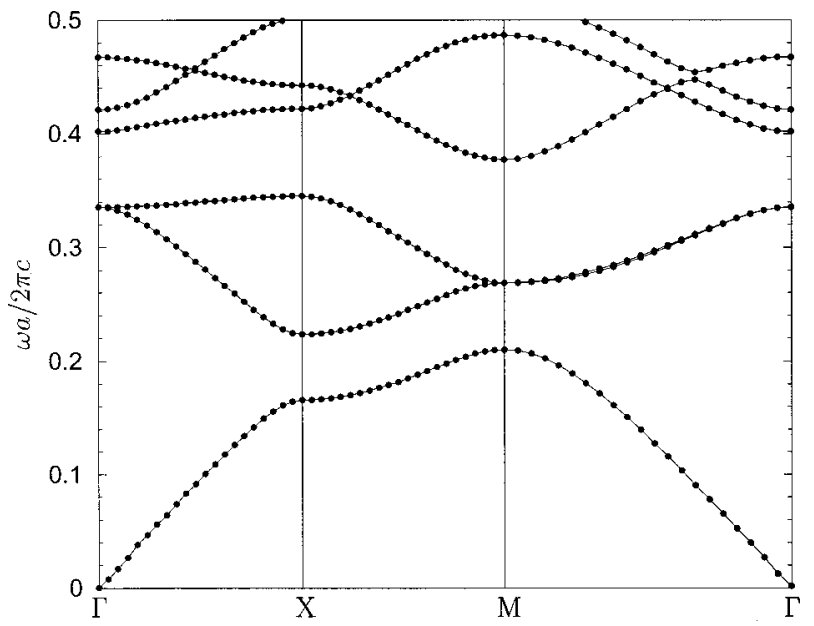

Fig. 1. Photonic band structure for $E$-polarized radiation in a 2-D photonic crystal consisting of a square array of dielectric cylinders $\left(r / a=0.4\right.$ and $\left.\epsilon_{c}=12\right)$ in air.

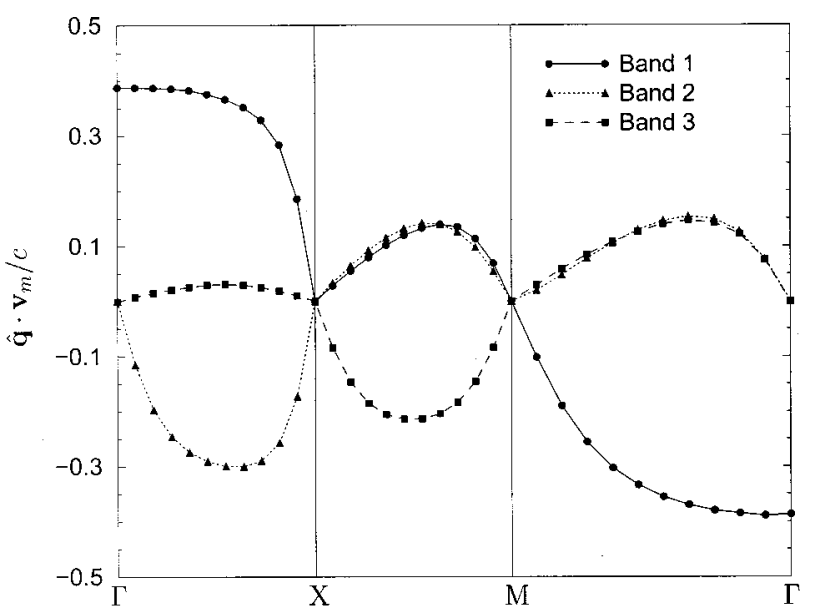

Fig. 2. Group velocities $\mathbf{q} \cdot \mathbf{v}_{m}$ for the three lowest bands for $E$-polarized radiation in a 2 -D photonic crystal consisting of a square array of dielectric cylinders. The photonic-crystal parameters are the same as for Fig. 1. 


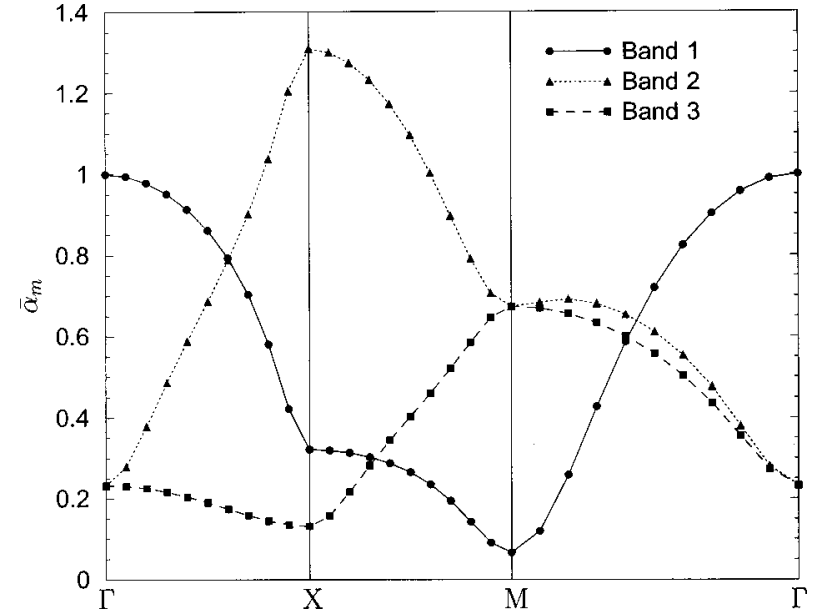

Fig. 3. Dimensionless gain-enhancement factor $\bar{\alpha}_{m}$ for the three lowest bands for $E$-polarized radiation in a 2 -D photonic crystal consisting of a square array of dielectric cylinders. The active medium occupies the space between the cylinders, and the photonic-crystal parameters are the same as for Fig. 1.

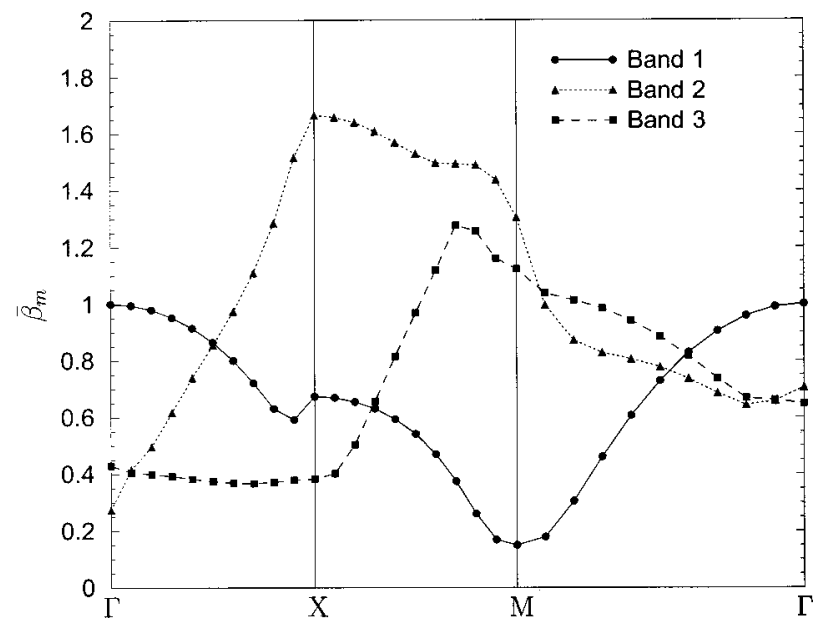

Fig. 4. Dimensionless saturation-enhancement factor $\bar{\beta}_{m}$ for the three lowest bands for $E$-polarized radiation in a 2 -D photonic crystal consisting of a square array of dielectric cylinders. The active medium occupies the space between the cylinders, and the photonic-crystal parameters are the same as for Fig. 1.

the group velocity $\left|\hat{\mathbf{q}} \cdot \mathbf{v}_{m}\right|$ (Fig. 2), and the scaled fieldenhancement factor $\bar{\alpha}_{m}$ (Fig. 3). From Fig. 4, we can see that the field enhancement of band 2 near the $X$ point, which is observed in Fig. 3, is accompanied by a corresponding enhancement of the effective nonlinear coupling. Because these enhancements offset each other in the slope, $\mathrm{d} I_{\text {output }} / \mathrm{d} R$, the input-output characteristic is dominated by the group-velocity contributions to $\gamma_{m}$ and the electromagnetic mode-density contribution to $I_{\text {sat }}$ and an enhancement of the slope is expected at the photonic band edge.

The spectral linewidth $\Delta \nu$ of a laser is given by the Schawlow-Townes formula ${ }^{24}$

$$
\Delta \nu=\frac{\Delta \nu_{c}}{n_{\mathrm{ss}}}
$$

where $\Delta \nu_{c}=\gamma_{m} / 2 \pi$ is the passive cavity bandwidth, dramatically reduced at the photonic band edge, and $n_{\mathrm{ss}}$ is the steady-state photon number. For the photonic crystal this steady-state number of photons is obtained from Eq. (49), which yields

$$
n_{\mathrm{ss}}=\frac{n_{\mathrm{sat}}^{0}}{\beta_{m}}\left(\frac{R}{R_{\mathrm{thr}}}-1\right),
$$

where $n_{\text {sat }}^{0}$ denotes the free-space saturation photon number and is independent of the photonic-crystal parameters. Clearly, the linewidth of the photonic-crystal laser is influenced not only by the modified threshold value of pumping $R_{\text {thr }}$ but also by the modified coupling parameter $\beta_{m}$.

\section{CONCLUSION}

Using a multiscale analysis, we have derived the generalized Maxwell-Bloch equations for laser action in a photonic crystal whose radiation modes interact with an incoherently pumped atomic system. This semiclassical model is formally equivalent to that of a laser in a uniform cavity with certain effective parameters related to features of the underlying photonic crystal. These parameters have clear physical meaning and can be calculated from the band structure and the associated Bloch modes of the photonic crystal. We have shown that, in a photonic crystal, the laser threshold can be modified significantly from that of a cavity containing a uniform dielectric by three independent factors: (i) the redistribution of field energy in a Bloch mode relative to that of a plane wave and the corresponding overlap of the Bloch mode with the light-emitting atoms, (ii) the decrease of the cavity-leakage rate caused by alterations in the group velocity of the electromagnetic mode in a photonic crystal, and (iii) changes in the spontaneous emission rate from active atoms related to the LDOS. We have shown that these same effects directly influence the input-output characteristics of the laser and the laser linewidth. In contrast to the laser threshold, the input-output characteristics and the linewidth are further influenced by an effective nonlinear coupling parameter $\beta_{m}$.

An interesting consequence of the above results is that laser action in photonic crystals containing active material with broad emission spectra, such as laser dyes, is likely to occur for frequencies near a photonic band edge. This is despite the fact that in 2-D or 3-D systems the density of states for frequencies near the band edge may be considerably smaller than it is when well within the allowed bands.

In our analysis, we have assumed that the photoniccrystal LDOS (in the frequency range of the atomic transition and in the vicinity of the light-emitting atom) is a smooth function of frequency over the frequency range spanned by the linewidth of the atomic transition. In strongly scattering photonic crystals and materials exhibiting a complete 3-D PBG this assumption may require reconsideration. This reconsideration may lead to further modification of laser characteristics associated with the non-Markovian radiative dynamics of the individual atoms. ${ }^{12}$ The results of our semiclassical analysis may 
be regarded as a starting point for the analysis of nonMarkovian radiative effects that are expected to arise near the band edges of more strongly scattering photonic crystals. Such effects may be addressed most accurately in the context of a fully quantized model of a photoniccrystal laser.

\section{ACKNOWLEDGMENTS}

L. Florescu acknowledges financial support from the Ontario Graduate Scholarship Program. K. Busch would like to thank U. Lemmer and A. Gombert for fruitful discussions and acknowledges the financial support by the Deutsche Forschungsgemeinschaft under grant Bu 1107/ 2-1 (Emmy-Noether program) and Bu 1107/3-1 (Schwerpunktprogramm Photonische Kristalle SP 1113). This study was supported in part by the Natural Sciences and Engineering Research Council of Canada.

The authors' e-mail addresses are as follows: L. Florescu, lucia@physics.utoronto.ca; K. Busch, kurt @tkm.physik.uni-karlsruhe.de; S. John, john@physics .utoronto.ca.

\section{REFERENCES}

1. C. M. Soukoulis, ed., Photonic Band Gap Materials, NATO ASI Series E (Kluwer, Dordrecht, The Netherlands, 1996) Vol. 315

2. C. M. Soukoulis, ed., Photonic Crystals and Light Localization in the 21st Century, NATO ASI Series C (Kluwer, Dordrecht, The Netherlands, 2001), Vol. 563.

3. S. John, "Electromagnetic absorption in a disordered medium near a photon mobility edge," Phys. Rev. Lett. 53, 2169-2172 (1984).

4. S. John, "Strong localization of photons in certain disordered dielectric superlattices," Phys. Rev. Lett. 58, 2486 2489 (1987)

5. E. Yablonovitch, "Inhibited spontaneous emission in solidstate physics and electronics," Phys. Rev. Lett. 58, 2059 2062 (1987)

6. S. John and J. Wang, "Quantum electrodynamics near a photonic band gap: photon bound states and dressed atoms," Phys. Rev. Lett. 64, 2418-2421 (1990).

7. H. Yokoyama and S. D. Brorson, "Rate equation analysis of microcavity lasers,” J. Appl. Phys. 86, 4801-4805 (1989).

8. G. Bjork and Y. Yamamoto, "Analysis of semiconductor microcavity lasers using rate equations," IEEE J. Quantum Electron. 27, 2386-2396 (1991).

9. S. John and T. Quang, "Collective switching and inversion without fluctuation of two-level atoms in confined photonic systems," Phys. Rev. Lett. 78, 1888-1891 (1997).

10. M. Florescu and S. John, "Single-atom switching in photonic crystals," Phys. Rev. A 64, 033801-1-033801-21 (2001).
11. S. John and M. Florescu, "Photonic band gap materials: toward an all-optical micro-transistor," J. Opt. A, Pure Appl. Opt. 3, S103-S120 (2001).

12. N. Vats and S. John, "Non-Markovian quantum fluctuations and superradiance near a photonic band edge," Phys. Rev. A 58, 4168-4184 (1998).

13. V. I. Kopp, B. Fan, H. K. M. Vithana, and A. Z. Genack, "Low-threshold lasing at the edge of a photonic stop band in cholesteric liquid crystals," Opt. Lett. 23, 1707-1709 (1998).

14. G. A. Turnbull, P. Andrew, M. J. Jory, W. L. Barnes, and I. D. W. Samuel, "Relationship between photonic band structure and emission characteristics of a polymer distributed feedback laser," Phys. Rev. B 64, 125122-1-125122-6 (2001).

15. M. Meier, A. Mekis, A. Dodabalapur, A. Timko, R. E. Slusher, J. D. Joannopoulos, and O. Nalamasu, "Laser action from two-dimensional distributed feedback in photonic crystals," Appl. Phys. Lett. 74, 7-9 (1999).

16. M. Imada, S. Noda, A. Chutinan, T. Tokuda, M. Murata, and G. Sasaki, "Coherent two-dimensional lasing action in surface-emitting laser with triangular-lattice photonic crystal structure," Appl. Phys. Lett. 75, 316-318 (1999).

17. J.-K. Hwang, H.-Y. Ryu, D.-S. Song, I.-Y. Han, H.-W. Song, H.-K. Park, Y.-H. Lee, and D.-H. Jang, "Room-temperature triangular-lattice two-dimensional photonic band gap lasers operating at $1.54 \mu \mathrm{m}$," Appl. Phys. Lett. 76, 2982-2984 (2000).

18. M. Meier, A. Dodabalapur, J. A. Rogers, R. E. Slusher, A. Mekis, A. Timko, C. A. Murray, R. Ruel, and O. Nalamasu, "Emission characteristics of two-dimensional organic photonic crystal lasers fabricated by replica molding," J. Appl. Phys. 86, 3502-3507 (1999).

19. S. Riechel, C. Kallinger, U. Lemmer, J. Feldmann, A. Gombert, V. Wittwer, and U. Scherf, "A nearly diffraction limited surface emitting conjugated polymer laser utilizing a two-dimensional photonic band structure," Appl. Phys. Lett. 77, 2310-2312 (2000).

20. J. P. Dowling, M. Scalora, M. J. Bloemer, and C. M. Bowden, "The photonic band edge laser: a new approach to gain enhancement," J. Appl. Phys. 75, 1896-1899 (1994).

21. S. Nojima, "Enhancement of optical gain in twodimensional photonic crystals with active lattice points," Jpn. J. Appl. Phys. Lett. 37, L565-L567 (1998).

22. K. Sakoda, K. Ohtaka, and T. Ueta, "Low-threshold laser oscillation due to group-velocity anomaly pelicular to twoand three-dimensional photonic crystals," Opt. Express 4, 481-489 (1999), http://epubs.osa.org/opticsexpress.

23. N. Susa, "Threshold gain and gain-enhancement due to distributed-feedback in two-dimensional photonic-crystal lasers," J. Appl. Phys. 89, 815-823 (2001).

24. A. E. Siegman, Lasers (University Science, Mill Valley, Calif., 1986).

25. A. Nayfeh, Perturbation Methods (Wiley, New York, 1973).

26. C. M. de Sterke and J. E. Sipe, "Envelope-function approach for the electrodynamics of nonlinear periodic structures," Phys. Rev. A 38, 5149-5165 (1988).

27. J. E. Sipe, "Vector $k \cdot p$ approach for photonic band structures,” Phys. Rev. E 62, 5672-5677 (2000).

28. D. Hermann, M. Frank, K. Busch, and P. Wölfle, "Photonic band structure computation," Opt. Express 8, 167-172 (2001), http://epubs.osa.org/opticsexpress.

29. M. Sargent III, M. O. Scully, and W. E. Lamb, Laser Physics (Addison-Wesley, Reading, Mass., 1977). 